$10.1117 / 2.1201208 .004447$

\title{
Color remapping turns night into day
}

\section{Alexander Toet}

A real-time mapping technique enables full-color rendering of multispectral nighttime images, making them as clear and colorful as daylight ones.

Night vision cameras are a vital source of information for a wide range of critical military and law enforcement applications such as surveillance, reconnaissance, intelligence gathering, and security. Currently, monochrome display of night imagery is still the standard. However, monochrome images often do not look natural, provide lower feature contrast, and they tend to induce visual illusions and fatigue. Intuitive color representations of night-vision imagery may alleviate these problems.

The increasing availability of multi-spectral, night-vision systems has led to a growing interest in the color display of night imagery. Color may improve feature contrast and reduce visual clutter, thus enabling better scene recognition, object detection, and depth perception. Most current techniques to colorize multi-band, night-time imagery are computationally expensive or do not yield natural and stable color settings. To resolve these issues, we developed a simple color remapping technique that provides colored night-time imagery with an intuitive and stable appearance. ${ }^{1}$ The method is computationally efficient and can easily be deployed in real-time.

Our color remapping technique assumes a fixed relation between false color tuples and natural color triplets for bands near the visual spectrum. This allows its implementation as a simple color-table swapping operation. For bands that are not correlated with the visual spectrum, color remapping can be used to enhance the detectability of targets through contrast enhancement and color highlighting.

We achieved color remapping by associating the multi-band sensor signal to an indexed false color image and swapping its color table with that of a regular daylight color image of a similar scene (see Figure 1). A wide range of environments can be represented with only a limited number of color tables. These tables need to be constructed only once before the system is deployed.

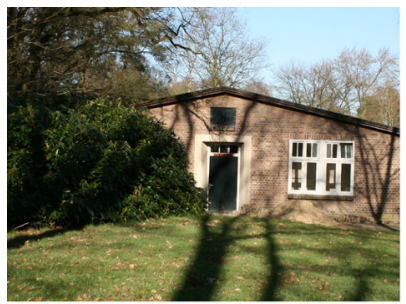

(a)

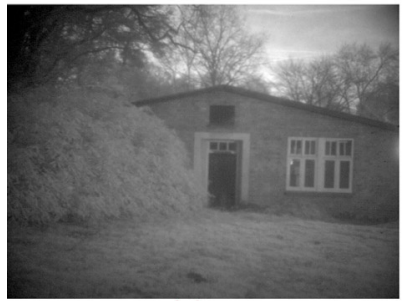

(c)
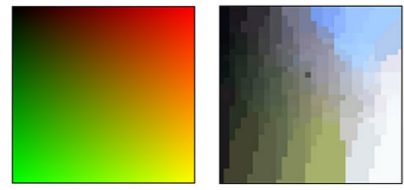

(e)

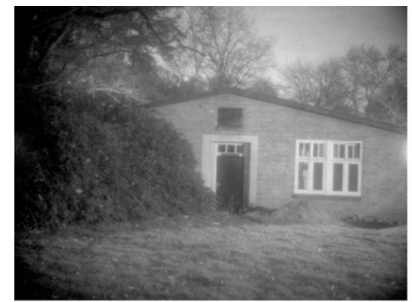

(b)

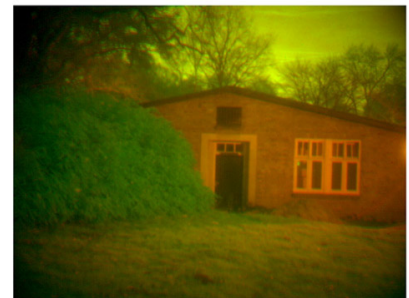

(d)

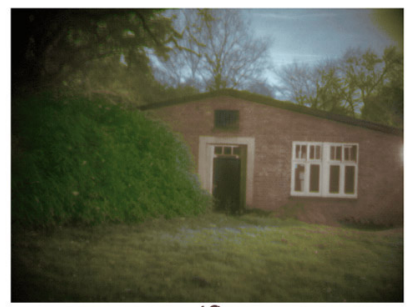

(f)
Figure 1. (a) Daylight color image, (b) visible, and (c) near-IR nighttime images of the same scene. (d) False color representation of $(b)$ and (c). (e) Color tables of (d) and (a). (f) Image (d) after color remapping.

The derivation of the color transformation requires a color photograph representing the intended operating theater or a similar, but not necessarily the same, environment. Then there are two options: either transfer the color statistics of this photograph to the false color multi-spectral image (when both images represent different but similar scenes), or establish a samplebased mapping between corresponding pixel values. ${ }^{1}$

To achieve an efficient real-time implementation, we used indexed, color-image representations and performed all required

Continued on next page 


\section{Newsroom}

(a)

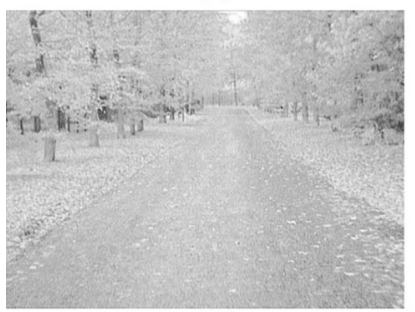

(c)

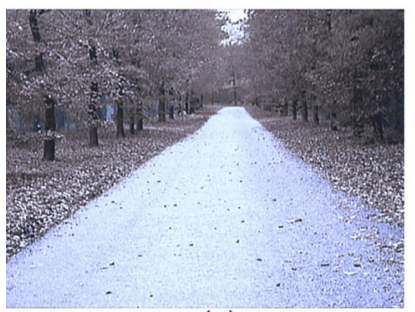

(e)

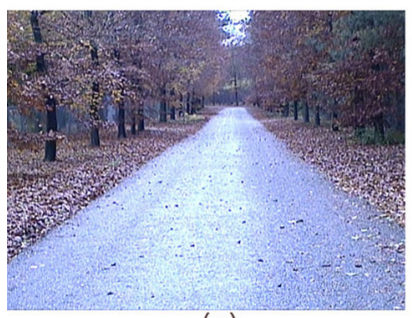

(g)

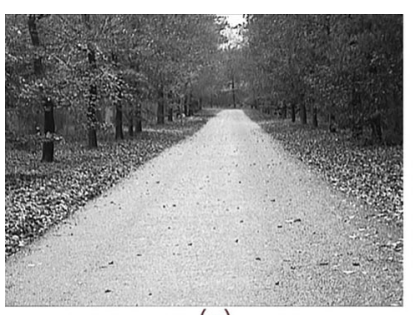

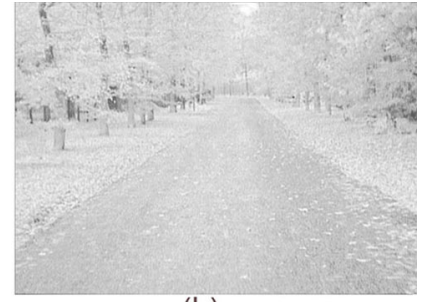

(b)

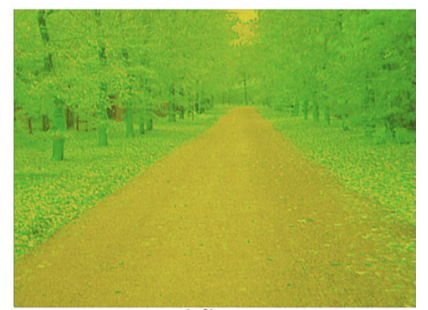

(d)

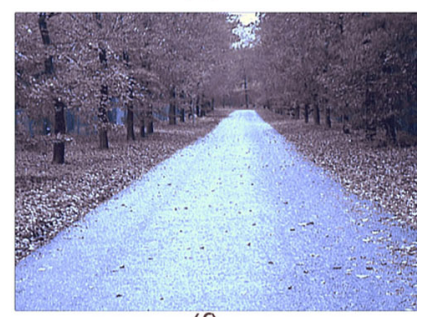

(f)

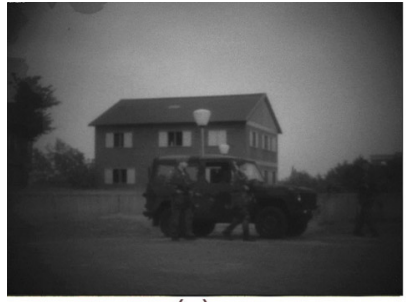

(a)

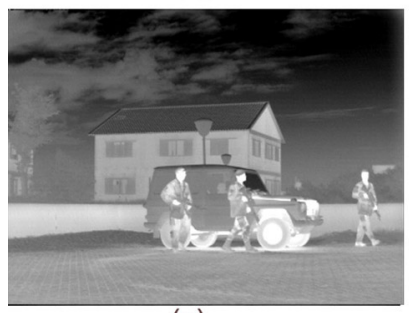

(c)

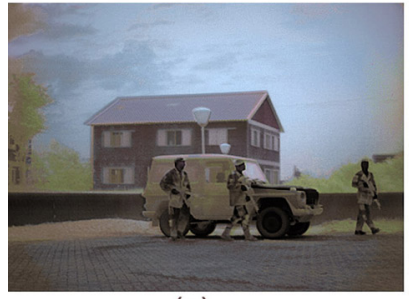

(e)

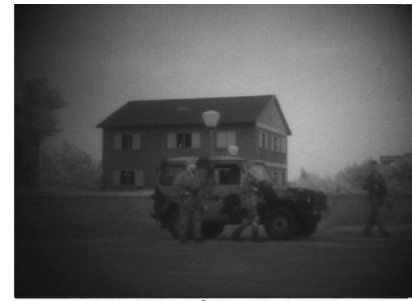

(b)

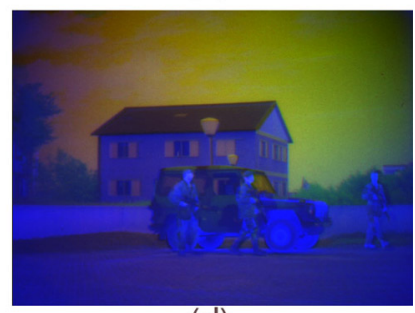

(d)

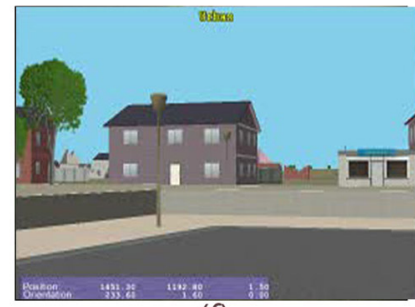

(f)
Figure 3. (a-c) Visual, near-IR, and long-wave IR night-time images of the same scene. (d) False color image obtained from (a-c). (e) Image (d) after replacing the color table of (d) by that of a corresponding synthetic scene (f).

evaluate a scene. ${ }^{7}$ Mappings emphasizing hot targets facilitate the detection of persons and vehicles in a scene. The inherent color constancy provided by the method gives dynamic imagery a stable appearance when the sensor suite pans over or moves through a scene. By integrating a multi-band, night-vision system with a surveillance and observation system that generates real-time, synthetic $3 \mathrm{D}$ environment views from a geometric $3 \mathrm{D}$ scene model, we were able to demonstrate that the use of synthetic imagery also serves to derive appropriate color mappings (see Figure 3). ${ }^{8}$

The color remapping procedure was initially developed to colorize multi-band, night-vision imagery. However, since the method can be applied to enhance specific image details, there are also law enforcement, surveillance, medical, and industrial applications. Going forward, we will use newly developed color image quality metrics to derive optimal mappings for each of these applications. portable real-time, multi-band night-vision systems ${ }^{2-4}$ that were deployed in several night-time field trials. ${ }^{5,6}$ It appears that color makes it significantly easier to distinguish more details and 


\title{
Author Information
}

\begin{abstract}
Alexander Toet
Netherlands Organization for Applied Scientific Research (TNO)
\end{abstract}

Soesterberg, The Netherlands

Alexander Toet received his $\mathrm{PhD}$ from Utrecht University, The Netherlands. He is currently a senior research scientist at TNO and has worked on image fusion for 25 years. His background is in visual perception and image processing.

\section{References}

1. A. Toet and M. A. Hogervorst, Progress in color night vision, Opt. Eng. 51 (1), pp. 010901-1-19, 2012. doi:10.1117/1.OE.51.1.010901

2. A. Toet and M. A. Hogervorst, Portable real-time color night vision, Proc. SPIE 6974, pp. 1-12, 2008. doi:10.1117/12.775405

3. A. Toet and M. A. Hogervorst, TRICLOBS portable triband low-light color observation system, Proc. SPIE 7345, pp. 1-11, 2009. doi:10.1117/12.817526

4. A. Toet, M. A. Hogervorst, J. Dijk, and R. van Son, INVIS: integrated night vision surveillance and observation system, Proc. SPIE 7689, pp. 1-16, 2010. doi:10.1117/12.849017

5. Website for video of the night-vision system. http://www.scivee.tv/ node/29094. Accessed 8 August 2012.

6. Website for video of the night-vision system. http://www.scivee.tv/ node/29095. Accessed 8 August 2012.

7. A. Toet, Cognitive image fusion and assessment, in O. Ukimura ed., Image Fusion, pp. 303-340, 2011. http://intechweb.org. Accessed 8 August 2012. doi: $10.5772 / 14787$

8. A. Toet, M. A. Hogervorst, R. van Son, and J. Dijk, Augmenting full-color fused multi-band, night-vision imagery with synthetic imagery for enhanced situational awareness, Int'1. J. Image and Data Fusion 2 (4), pp. 287-308, 2011. doi:10.1080/19479832.2011.598135 